\title{
Cierre percutáneo de comunicación interauricular en paciente con peso menor de 15 kilogramos utilizando técnica asistida con balón. Reporte de un caso
}

\author{
Percutaneous closure of atrial septal defect in patients under 15 kilograms using balloon \\ assisted technique. Case report
}

\author{
Guillermo Aristizabal-Villa ${ }^{1 *}$, Alexis Plata-Marriaga ${ }^{2}$ y Jorge Escobar-Modesto ${ }^{3}$ \\ ${ }^{1}$ Departamento de Cardiologia Pediátrica y Hemodinamia, Clínica de la Costa, Barranquilla; 2Departamento de Investigación, Clínica Madre \\ Bernarda, Cartagena; ${ }^{3}$ Departamento de Pediatría, Clínica de la Costa, Barranquilla. Colombia
}

\section{Presentación del caso}

Niña de 3 años, con peso de $14 \mathrm{~kg}$ y antecedentes de episodios de neumonía adquirida en la comunidad y comunicación interauricular. En la exploración física se encuentra a la auscultación un soplo sistólico de grado II/IV en foco pulmonar y desdoblamiento del segundo ruido cardiaco. La radiografía de tórax muestra un índice cardiotorácico de 0.6 con hiperflujo pulmonar. En el electrocardiograma se observa eje cardiaco a la derecha y bloqueo incompleto de rama derecha. Se realiza ecocardiograma transtorácico en el que se evidencia comunicación interauricular tipo ostium secundum de $14 \mathrm{~mm}$ de diámetro con cortocircuito de izquierda a derecha, dilatación importante del ventrículo derecho y arteria pulmonar. Se evalúan los bordes que rodean el defecto, encontrando un borde superior de $10 \mathrm{~mm}$, un borde inferior de $8 \mathrm{~mm}$ y un borde posteroinferior de $9 \mathrm{~mm}$, con ausencia de borde anterior (en relación con la aorta). Se decide el cierre percutáneo del defecto por los datos de repercusión hemodinámica. Durante el registro hemodinámico con oxígeno al $21 \%$ se encontró una presión media de la arteria pulmonar de $18 \mathrm{mmHg}$, salto oximétrico en la aurícula derecha, Qp/Qs 3.9/1 y resistencia vascular pulmonar indexada de 0.8 unidades Woods por metro cuadrado de superficie corporal. En el diagnóstico angiográfico no se encontraron otros cortocircuitos. Con ayuda de ecocardiograma transesofágico se evalúa el tabique interauricular, encontrando comunicación tipo ostium secundum de 14 $\mathrm{mm}$ de diámetro y corroborando la ausencia de borde anterior (aórtico). Previo a la administración de heparina $(100 \mathrm{U} / \mathrm{kg})$ y registro del tiempo de coagulación activado (>250 s), en proyección angiográfica posteroanterior, se intenta el cierre del defecto con un dispositivo Occlutech Figulla Flex II $18 \mathrm{~mm}$. Se observó luxación del disco izquierdo del dispositivo sobre la aorta, hacia la aurícula derecha, debido a la ausencia de soporte del borde anterior del defecto. Se consideró entonces dar soporte al dispositivo con la técnica asistida con balón. Se obtuvo otro acceso venoso femoral izquierdo y se posicionó la guía de soporte en la vena pulmonar superior izquierda, avanzando el balón de medición de 25 mm (Occlutech Sizing BaIloon), posicionándolo a través del defecto septal e insuflándolo de manera parcial. Se procedió a liberar el disco izquierdo en la aurícula izquierda (Fig. 1), retrayendo en conjunto el dispositivo y el sistema

\section{Correspondencia:}

*Guillermo Aristizabal-Villa

E-mail: Guillermo.aristizabal68@gmail.com
Disponible en internet: $20-10-2020$ Arch Cardiol Mex. 2021;91(2):261-263 www.archivoscardiologia.com CC BY-NC-ND (http://creativecommons.org/licenses/by-nc-nd/4.0/).

Fecha de recepción: 28-06-2020

Fecha de aceptación: 21-08-2020 DOI: 10.24875/ACM.20000322 


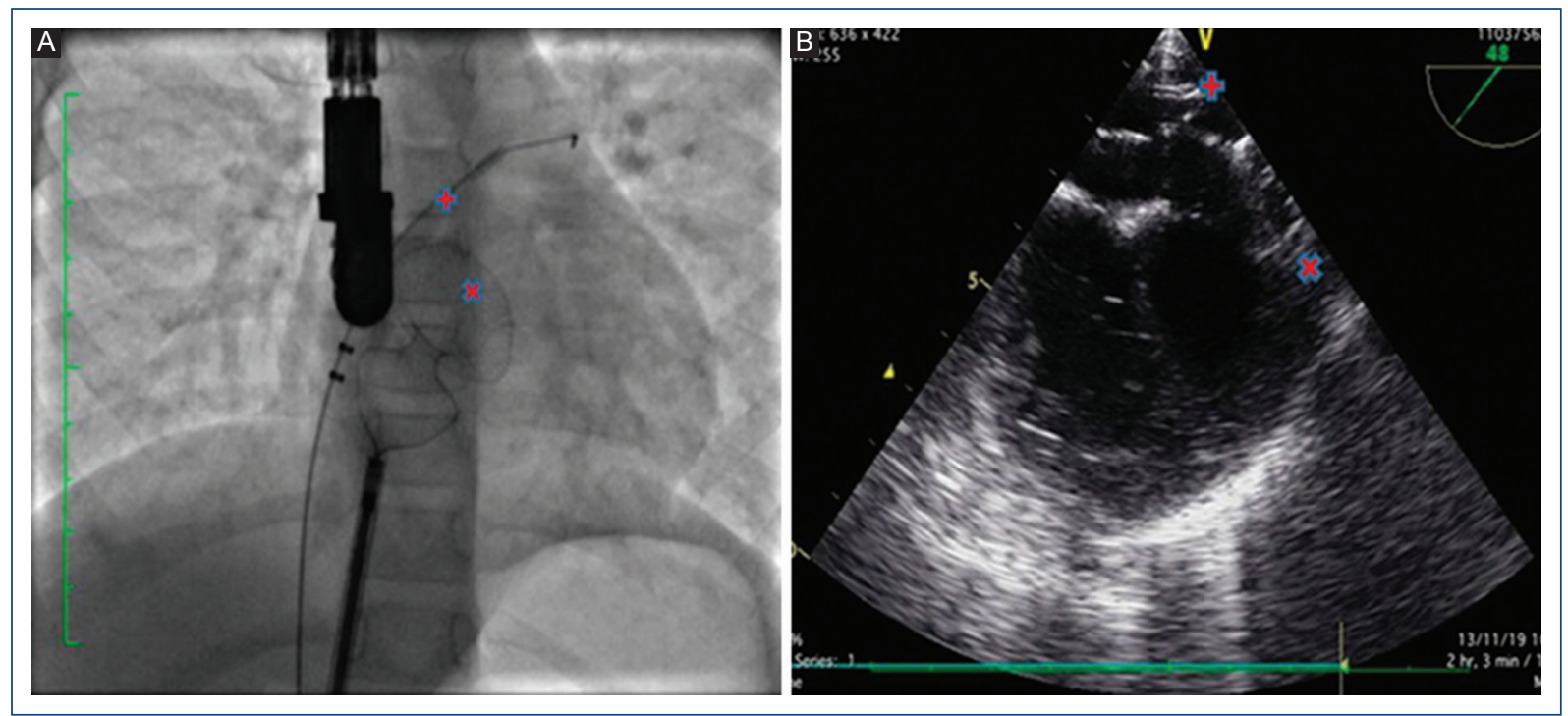

Figura 1. A: imagen fluoroscópica. B: ecocardiografía transesofágica simultánea. Se observa el dispositivo oclusor de la comunicación interauricular desplegándose $(x)$. Se usa el balón de medición parcialmente inflando $(+)$ para dar soporte al disco izquierdo del dispositivo, manteniendo su posición en la aurícula izquierda y evitando de esta forma su desplazamiento o luxación hacia la aurícula derecha.

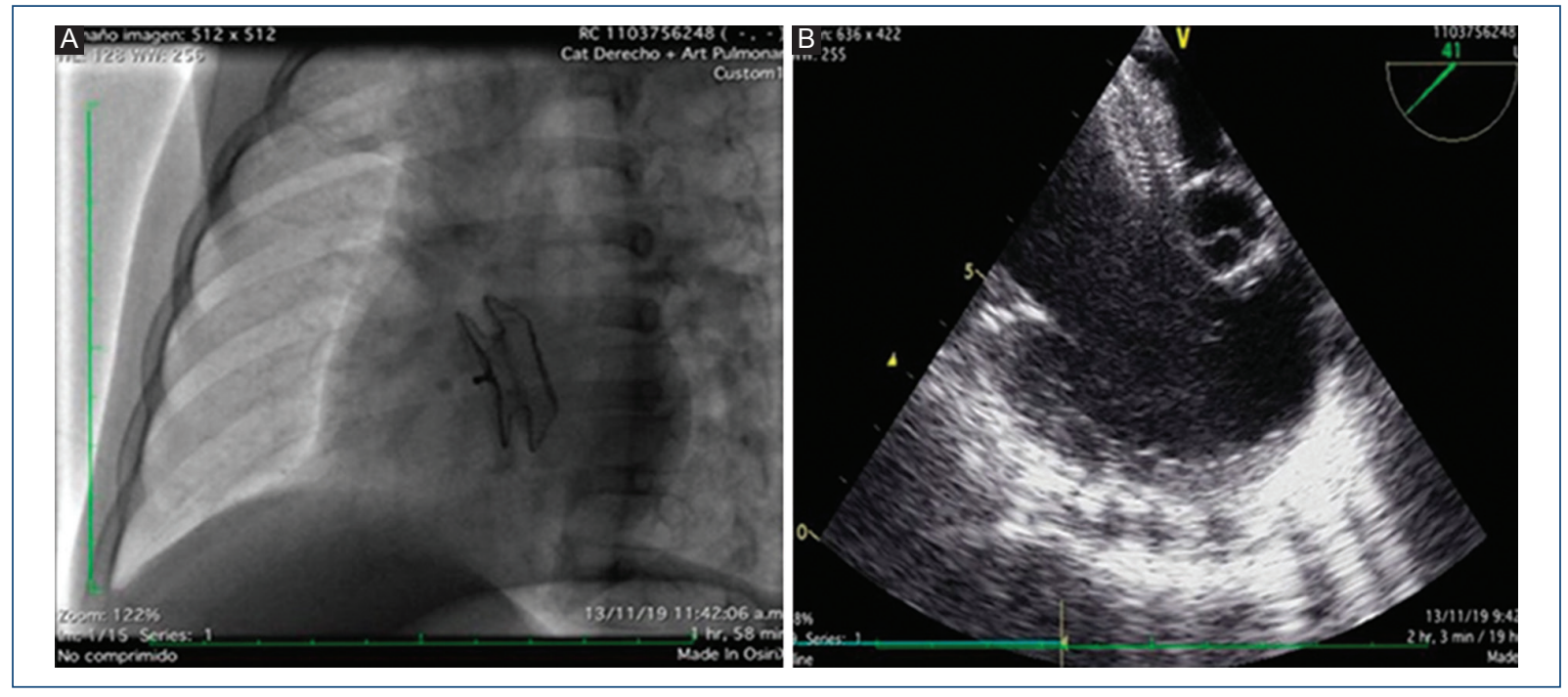

Figura 2. A: Imagen fluoroscópica. B: ecocardiografía transesofágica simultánea. Se observa el dispositivo oclusor de la comunicación interauricular liberado con adecuada implantación sobre el tabique interauricular (B), con una separación óptima de los discos (A).

liberador hacia el tabique interauricular, liberando el disco derecho en la aurícula derecha sin observar luxación del disco izquierdo. Se desinfló y retiró el balón, y se liberó el dispositivo sin complicaciones (Fig. 2). La paciente no presenta ninguna alteración electrocardiográfica durante la intervención. El tiempo de procedimiento fue de 60 minutos. No se requirió dosis adicional de heparina. A las 24 horas del procedimiento se realizó ecocardiograma transtorácico, encontrando el dispositivo en adecuada posición, sin desplazamientos y sin derrame pericárdico. Se dio de alta a la paciente a las 24 horas con esquema de ácido acetilsalićlico y clopidogrel durante 6 y 2 meses, respectivamente. No se encontraron alteraciones 
electrocardiográficas ni ecocardiográficas al mes y a los 6 meses de seguimiento.

La mayoría de los niños con comunicación interauricular son asintomáticos o presentan síntomas leves, por lo que el cierre percutáneo se tiende a aplazar hasta los 4 años de edad o con un pesos superior a $15 \mathrm{~kg}$; lo anterior, debido a la alta tasa de cierre espontáneo de los defectos menores de $8 \mathrm{~mm}$ en los primeros años de vida ${ }^{1,2}$. Algunos pacientes con gran cortocircuito de izquierda a derecha presentan falla de medro, infecciones de vías respiratorias bajas de repetición o episodios de crisis asmáticas con múltiples hospitalizaciones, por lo que estaría indicado el cierre del defecto a pesar del peso y la edad principalmente en aquellos con antecedente de prematuridad o cromosomopatías. Los defectos interauriculares en este grupo de pacientes se asocian a una cavidad auricular izquierda mas pequeña, lo que resulta en mayor dificultad para posicionar el disco izquierdo, generando un mal alineamiento con el tabique interauricular $y$ luxación del dispositivo a través del defecto. Por lo anterior, la técnica de cierre habitual tiende a fallar en la mayoría de los casos. Sharma, et al. ${ }^{2}$ publicaron un estudio con 45 pacientes pediátricos, con una media de 25 meses edad y un peso promedio de $8.9 \mathrm{~kg}$, en los que la técnica de liberación habitual solo fue efectiva en 15 pacientes, requiriendo maniobras especiales de liberación 28 pacientes; la más frecuentemente usada fue la liberación en la vena pulmonar superior izquierda, con 18 casos, seguida por la técnica asistida con balón en seis casos, la liberación en la vena pulmonar superior derecha en tres casos y la liberación en la orejuela izquierda en un caso. La técnica asistida con balón tiene la desventaja de requerir otro acceso vascular venoso, un operador adicional para sostener el balón, y mayor tiempo de procedimiento y de hemostasia ${ }^{3}$. Se ha demostrado la eficacia del cierre del defecto septal en pacientes de bajo peso, con mejoría de los síntomas y del peso para la edad en el seguimiento a largo plazo, así como regresión en los datos ecocardiográficos de sobrecarga de volumen del ventrículo derecho ${ }^{4}$. En cuanto a la seguridad del procedimiento, el riesgo de complicaciones mayores y menores es comparable con el de los pacientes de mayor edad y peso, teniendo en cuenta que los pacientes con edad menor y bajo peso presentan comorbilidad que se asocia con prolongada estancia hospitalaria ${ }^{5}$.
Como conclusiones, el cierre percutáneo de defectos septales interauriculares en pacientes con bajo peso 0 edad tiene indicaciones precisas, como falla de medro, infecciones respiratorias de repetición asociado a prematuridad o cromosomopatías. La técnica habitual de cierre percutáneo en este tipo de pacientes es fallida en un alto porcentaje, debido al menor tamaño de la aurícula izquierda que no permite acomodar el dispositivo sobre el tabique interauricular. Se deben implementar maniobras especiales de liberación, con las cuales se puede llevar a cabo el cierre del defecto de forma segura y eficaz.

\section{Conflicto de intereses}

Ninguno.

\section{Financiamiento}

La presente investigación no ha recibido ninguna beca específica de agencias de los sectores público, comercial o sin ánimo de lucro.

\section{Responsabilidades éticas}

Protección de personas y animales. Los autores declaran que para esta investigación no se han realizado experimentos en seres humanos ni en animales.

Confidencialidad de los datos. Los autores declaran que han seguido los protocolos de su centro de trabajo sobre la publicación de datos de pacientes.

Derecho a la privacidad y consentimiento informado. Los autores declaran que en este artículo no aparecen datos de pacientes.

\section{Bibliografía}

1. Vogel M, Berger F, Dähnert I, Ewert P, Lange PE. Treatment of atrial septal defects in symptomatic children aged less than 2 years of age using the Amplatzer septal occluder. Cardiol Young. 2000;10:534-7.

2. Sharma B, Pinto R, Dalvi B. Transcatheter closure of atrial septal defect in symptomatic children weighing $\leq 10 \mathrm{~kg}$ : addressing unanswered issues from a decade of experience. Ann Pediatr Cardiol 2020;13:4-10.

3. Dalvi B. Balloon assisted technique for closure of large atrial septal defects. Images Paediatr Cardiol. 2008;10:5-9.

4. Wyss Y, Quandt D, Weber R, Stiasny B, Weber B, Knirsch W, et al. Interventional closure of secundum type atrial septal defects in infants less than 10 kilograms: indications and procedural outcome. J Interv Cardiol. 2016;29:646-53.

5. Tangho G, Odermarsky M, Naumburg E, Liuba P. Early complications after percutaneous closure of atrial septal defect in infants with procedural weight less than $15 \mathrm{~kg}$. Pediatr Cardiol. 2017;38:25563. 